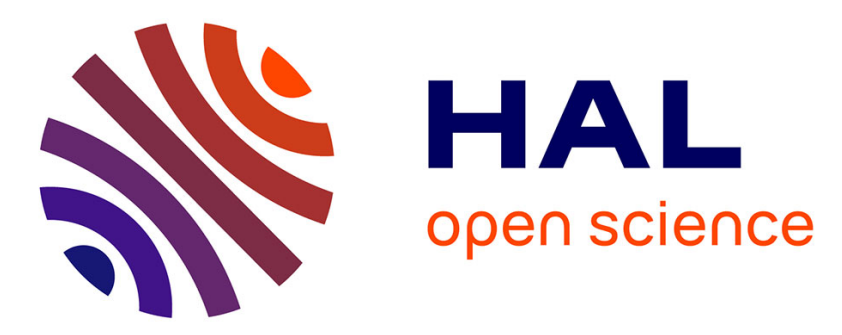

\title{
TOA Estimation for Positioning With DVB-T Signals in Outdoor Static Tests
}

Liang Chen,, Olivier Julien, Paul Thevenon, Damien Serant, Axel Javier Garcia Peña, Heidi Kuusniemi

\section{- To cite this version:}

Liang Chen,, Olivier Julien, Paul Thevenon, Damien Serant, Axel Javier Garcia Peña, et al.. TOA Estimation for Positioning With DVB-T Signals in Outdoor Static Tests. IEEE Transactions on Broadcasting, 2015, PP (99), 10.1109/TBC.2015.2465155 . hal-01205229

\section{HAL Id: hal-01205229 \\ https://hal-enac.archives-ouvertes.fr/hal-01205229}

Submitted on 29 Sep 2015

HAL is a multi-disciplinary open access archive for the deposit and dissemination of scientific research documents, whether they are published or not. The documents may come from teaching and research institutions in France or abroad, or from public or private research centers.
L'archive ouverte pluridisciplinaire HAL, est destinée au dépôt et à la diffusion de documents scientifiques de niveau recherche, publiés ou non, émanant des établissements d'enseignement et de recherche français ou étrangers, des laboratoires publics ou privés. 


\title{
TOA Estimation for Positioning with DVB-T Signals in Outdoor Static Tests
}

\author{
Liang Chen, Olivier Julien, Paul Thevenon, Damien Serant, Axel Garcia Peña \\ and Heidi Kuusniemi
}

\begin{abstract}
Digital television (DTV) signal is a promising signal of opportunity (SoO) for wireless positioning. This paper studies the time of arrival (TOA) estimation on the European standard DVB-T signals in the context of positioning. Theoretical analysis on the autocorrelation of the pilot signal and multipath error envelop (MEE) suggests that DVB-T has the appropriate characteristics for positioning. A software defined radio (SDR) based DVB-T receiver is developed for field testing, in which the radio frequency (RF) DVB-T signals are first sampled by USRP (Universal Software Radio Peripheral), and then algorithms of the coarse symbol synchronization, the pilot detection, the first path acquisition and the delay tracking are sequentially performed to obtain the TOA estimation. To quantify the TOA tracking results on real DVB-T signals, field testing campaigns are carried out in five cities in Southern France. Test results showed that, in the high signal-noise ratio (SNR) condition, the 2- $\sigma$ error interval is within 1 meter. By taking into account all the field tests, the interval of $95 \%$ accuracy is within $4 \mathrm{~m}$ and the corresponding $C / N_{0}$ varies from 48 to $62 \mathrm{~dB}-\mathrm{Hz}$. In addition, with the developed TOA estimation method, the synchronization of the emitters with GPS (Global Positioning System) time is evaluated and the distance difference of the multiple emitters within single frequency networks (SFNs) are also measured during the tests.
\end{abstract}

\section{Index Terms}

This work was supported in part by WIPINGDTV project (No. 254232) from Academy of Finland. Liang Chen is with the Department of Navigation and Positioning, Finnish Geospatical Research Institute (FGI), Geodeetinrinne 2 PL 15, 02431 Masala, Finland, e-mail:liang.chen@nls.fi. Olivier Julien, Paul Thevenon, Axel Garcia Peña are with SIGNAV Laboratory in École Nationale de L'Aviation Civile, 7 Avenue Edouard Belin, BP 54005, 31055 Toulouse CEDEX 4, France. Email: ojulien@ recherche.enac.fr, thevenon@ recherche.enac.fr., garcia-pena@recherche.enac.fr. Damien Serant is with the Navigation Department of Thales Alenia Space, France. Email: damien.serant@thalesaleniaspace.com. Heidi Kuusniemi is with the Department of Navigation and Positioning, Finnish Geospatical Research Institute (FGI), Geodeetinrinne 2 PL 15, 02431 Masala, Finland, Email: heidi.kuusniemi@nls.fi 
digital TV, wireless positioning, Orthogonal Frequency Division Multiplexing(OFDM), software defined radio (SDR), time of arrival, delay locked loop

\section{INTRODUCTION}

Recently, digital broadcasting systems, such as Digital Video Broadcasting (DVB), Digital Audio Broadcasting (DAB), and the ATSC (Advanced Television Systems Committee standards), have been widely deployed as an information transmission technique [1]. It has been recognized that novel wireless location methods can be designed by utilizing the digital broadcasting signals [2]. Compared with the GNSS (Global Navigation Satellite Systems), localization awareness based on the digital broadcasting has a range of potential advantages: the signal transmission power is stronger and the frequency band is within $300-900 \mathrm{MHz}$, which contribute to better urban propagation and building penetration than the currently used GPS L1 or Galileo E1 of around 1.5 GHz. Therefore, a better receiving quality indoors is expected [3]. The nominal signal bandwidth of the terrestrial digital TV broadcasting is designed between $6-8 \mathrm{MHz}$, which is much larger than the chipping rate of GPS L1 or Galileo E1 signals. This will improve the precision of the signal synchronization, and hence the accuracy of the derived pseudo-range measurements. The digital TV (DTV) signals are transmitted continuously and the location of the DTV transmitters are fixed. In contrast to the GNSS, the range between the DTV transmitters and the receivers changes very slowly. Therefore, the DTV signal does not experience significantly the Doppler effects and the impairment caused by the delay of ionosphere propagation, which will lead to easier signal acquisition and the possibility of integration over a longer period of time [2]. As a signal of opportunity (SoO) for positioning, the DTV facilities have already been deployed and no more infrastructure investment is required, except the position devices.

Based on these facts, research interest in localization awareness using DTV systems has grown rapidly. Basically, these methods can be divided into two categories: methods for single-carrier ATSC system [2], [4], [5] and methods for multicarrier Orthogonal Frequency Division Multiplex (OFDM) system [6], [7], [8], [9], [10], [11], [12], [13], [14], [15].

The results in reference [16] have showed that the location accuracy could reach meterscale with the ATSC DTV signals. However, the ATSC DTV is a single-carrier modulation system, which is vulnerable to multipath fading. In order to mitigate the effect of multipath fading for making the technique suitable for mobile application [17], digital broadcasting signals based on Orthogonal Frequency Division Multiplex (OFDM) modulation have been 
investigated and tested. As the standards based on OFDM modulation, e.g. DVB-T/H, T$\mathrm{DMB}$, etc, have widely been adopted in most countries, we may predict that the wireless position systems based on the multi-carrier OFDM have massive amounts of potential users in the future.

For the purpose of high accuracy positioning and navigation with the OFDM signals, there are several methods to determine the time of arrival (TOA) proposed in the literature. One of them is based on a sliding correlator for the coarse timing acquisition by using the property of OFDM cyclic prefix [18]. To achieve a finer time delay estimation, Delaylocked loop (DLL) or early-minus-late (EML) loop is used in [19]. Scatter pilots [6], [7], [8] or full OFDM symbols demodulated signal from the receivers [12] have been exploited as the local template in the DLL, which are the variants to the process inside conventional GNSS receivers. Time delay can also be obtained using the time-domain synchronous OFDM signals [10] or the transmitter signature waveforms [11].

In this paper, we will focus on the time of arrival (TOA) estimation and tracking with DVB-T signals. A complete software defined radio (SDR) receiver for TOA estimation is developed. Outdoor static testing campaigns are carried out to quantify the TOA tracking results. As an extension to the tests, the synchronization of the emitter is surveyed and the multiple emitters within the single frequency networks (SFN) are observed.

The paper is organized as follows: Section II briefly describes the DVB-T standard in the perspective of wireless positioning and presents the signal model with channel impairments. Section III describes the schemes of the TOA estimation. Section IV derives the ideal autocorrelation function used in our method and also analyzes the TOA tracking errors in theory. Section V describes the test bench used for sampling the real DVB-T signals. Outdoor experiments are described and test results are discussed in Section VI. Finally, in Section VII, conclusions are summarized.

\section{DVB-T STANDARD AND THE SIGNAL MODEL}

\section{A. Overview of ETSI DVB-T standard from the perspective of wireless positioning}

The DVB-T system, adopted as an European standard for digital TV terrestrial broadcasting [17], uses the OFDM (orthogonal frequency division multiplexing) modulation to achieve robust transmission in multipath scenarios. In DVB-T, OFDM signals are specified by three 
parameters: the number of subcarriers or the FFT (fast Fourier transform) size, the length of the cyclic prefix (CP) and the sampling period. There are several options/modes provided by the ETSI standard [17] to set up the parameters. In this study, all the field testing campaigns are carried out in Southern France, where the DVB-T parameters used are given in Table I.

From the perspective of wireless positioning, there are several properties in the DVB-T system that could be utilized. Firstly, DVB-T signals are organized in frames and transmitted in streams. More specifically, every OFDM frame consists of 68 OFDM symbols and every four frames constitute one super-frame. Besides, each OFDM symbol is transmitted with a fixed duration. Therefore, the DVB-T signals with continuous transmission and in a fixed rate will enable the receiver to continuously track the arrival of signal, which will improve the accuracy of the timing-based estimation for positioning.

Secondly, in each OFDM symbol, the pilot subcarriers, which are designed mainly for synchronization, channel estimation and equalization, are given by a known Pseudo-Random Binary Sequence (PRBS) and have the amplitudes boosted by a factor $\frac{4}{3}$ compared to the amplitude of the payload data or Transmission Parameter Signaling (TPS) symbols. Thus, by exploiting the autocorrelation property of the PRBS and the boosted power, it is promising to use pilots to achieve the accurate delay estimation in wireless positioning.

Lastly, it is also noted that, in DVB-T systems, multiple emitters are suggested to be coordinated to the GPS time and transmit the same DVB-T signals in the same frequency simultaneously, forming what is called SFN transmission. The advantage of the SFN is its ability to efficiently utilize the frequency resource, decrease the outage probability and increase the coverage area through the use of spatial diversity schemes at the receiver [20]. Meanwhile, for the purpose of wireless positioning, the network synchronization among the multiple emitters makes the timing-based estimation (TOA/TDOA) available. Figure 1 illustrates the SFN transmission of the DVB-T signals.

\section{B. Signal model}

Let us assume a DVB-T system consists of $N$ subcarriers, among which $N_{u}$ subcarriers at the central spectrum are used for transmission and the other subcarriers at both edges form the guard bands. Let $\left\{c_{n} \mid n=0, \cdots, N_{u}-1\right\}$ denote the modulated data or subcarrier symbol, where $n$ represents the subcarrier number After the iFFT (inverse fast Fourier transform) operation and by adding the guard interval for every OFDM symbol, the samples of the 
TABLE I

DVB-T PARAMETERS IN FRANCE

\begin{tabular}{lr}
\hline parameters & value \\
\hline FFT size (mode) & $8 \mathrm{k}(8192)$ \\
approximate bandwidth & $8 \mathrm{MHz}$ \\
CP ratio & $1 / 8$ \\
number of information carriers & 6817 \\
modulation of payload information & $64 \mathrm{QAM}$ \\
symbol duration $T_{s}$ & $1.008 \mathrm{~ms}$ \\
\hline
\end{tabular}

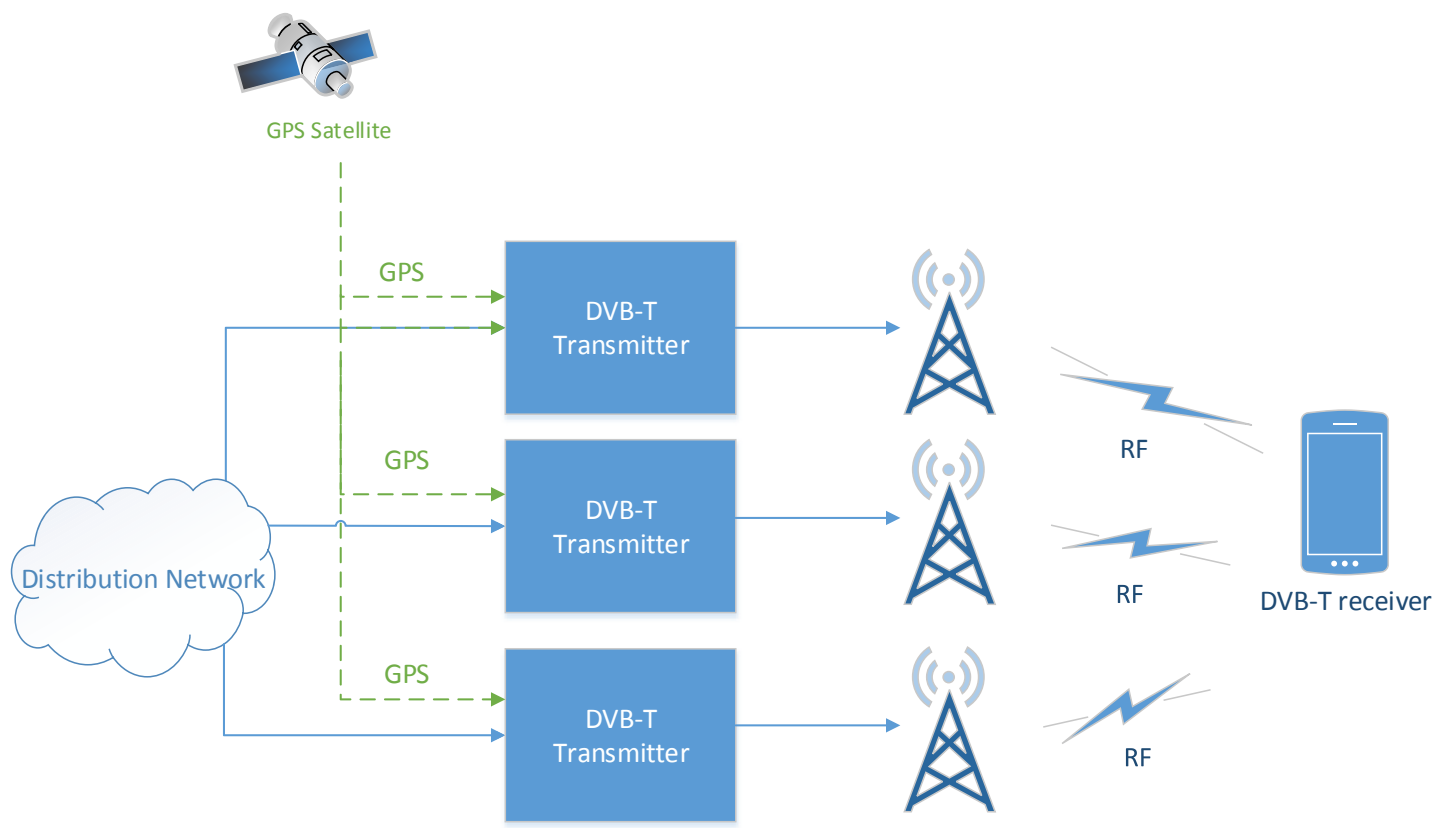

Fig. 1. DVB-T SFN transmission

transmitted baseband signal can be expressed as

$$
s(k)=\frac{1}{\sqrt{N}} \sum_{n=0}^{N_{u}-1} c_{n} e^{j 2 \pi k n / N} \quad-N_{g} \leq k \leq(N-1)
$$

where $N_{g}$ is the number of guard samples, and $j=\sqrt{-1}$. In (1), we assume the ideal Nyquist pulse shaping, which is realistic due to the presence of guard bands on the edge of the transmitted DVB-T signal's spectrum, the transceiver pulse shaping filters would have very little influence on the useful part of the spectrum located in the middle of the band.

As a typical wireless transmission system, DVB-T signals usually travel along multiple 
paths, arising from reflection, scattering and diffraction, which are due to numerous obstacles in the propagation environment. In ETSI DVB-T standard [17], two channel models are suggested for the terrestrial broadcasting transmission, i.e. a Rice channel model (F1), which is derived from the receivers with fixed position and a Rayleigh channel model (P1), the statistics of which is obtained from the receivers moving with slow speed. Both models have 20 multipaths, while the Rice model has an extra LOS component. Here, we assume that the signal is transmitted over a frequency selective fading channel of length $L$ with complex path gains $\left\{h_{l}\right\}$ and the corresponding path delays $\left\{\tau_{l}\right\}$, where $\{l=0,1, \cdots, L\}$.

At the receiver, there generally exist symbol-timing offset (STO), carrier-frequency offset (CFO), and sampling clock offset (SCO). To be more specific, STO corresponds to the time difference between the supposed and the real beginning of one OFDM symbol. CFO is caused by the mismatch of the receiver local oscillator frequency with the carrier of the received signal. SCO is due to the mismatch of the sampling clock frequency between transceiver and the Doppler effect. Hence, the received samples can be written as

$$
r(k)=e^{j(2 \pi k \Delta f / N+\varphi)} \Sigma_{l=0}^{L-1} h_{l} s\left(k-\tau_{l}\right)+n(k)
$$

where $n(k)$ is the sample of zero-mean complex Gaussian noise process with variance $\sigma^{2}$, $\Delta f$ is the CFO normalized by the subcarrier spacing and $\varphi$ is an arbitrary carrier phase factor. The timing point of the start of the FFT window is determined by the timing synchronization to be at the sample $r(\epsilon)$, where $\epsilon$ is a timing offset in units of OFDM samples.

It has been recognized that, the channel effect and the timing/frequency errors in (2) have important impact on the signal demodulation [21]. For the purpose of communications, the DTV receivers extract the timing measurements and recover the frequency offset from the received signals, which results in a synchronization problem. Although the multicarrier communication systems has stringent requirements on the timing and frequency synchronization for reliable communications, as the desired for positioning and navigation purpose, it is still necessary to get a finer synchronization which results in a finer time delay estimation of the received signals. In what follows, we will described the scheme for TOA estimation of the DVB-T signals, which is required for a highly accurate wireless positioning.

\section{TOA ESTIMATION FOR DVB-T POSITIONING}




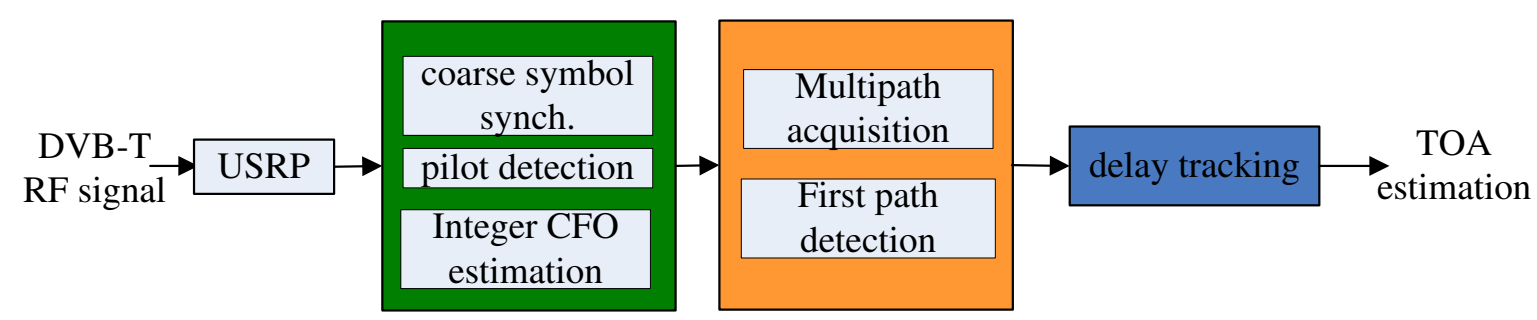

Fig. 2. Block diagram of the SDR DVB-T receiver for TOA estimation

In principle, the TOA estimation for DVB-T positioning is to find the starting time of the OFDM symbols as accurately as possible. Considering the time and frequency errors and the multipath effect mentioned in (2), three steps are carried out for DVB-T TOA estimation, namely the coarse synchronization and pilot detection, the first path acquisition and the delay tracking. In the coarse synchronization, the start of the OFDM symbol is estimated in the accuracy of one sample period. However, due to the various offsets and the multipath, the estimated timing errors could be as large as tens of samples. The step of the first path acquisition aims at detecting the multipath and acquire the first path of arrival within a fraction of one sample period. With the delay tracking, the TOA estimation is able to be achieved even more accurately. Figure 2 shows the block diagram of the receiver. The whole method will be described in detail as follows.

\section{A. coarse synchronization and pilot detection}

This step aims at coarse timing and fractional CFO estimation, detecting the pattern of the scattered pilot sequence and the integer CFO detection. For these purposes, the following algorithms are used in the developed receiver:

1) coarse symbol synchronization: The coarse symbol synchronization is to find the correct position of the receive DFT (discrete Fourier transform) window. Since the CFO is unknown at this stage, it is desirable that the timing recovery scheme be robust against possibly large frequency offset. In this study, we use the correlation method based on the CP for the coarse symbol synchronization [18]. Specifically, the time index of the sample with the maximum auto-correlation of the received signal is found by:

$$
\begin{aligned}
\Phi(k) & =\Sigma_{m=0}^{N_{\mathrm{CP}}-1} r(m+k) \cdot r^{*}(m+k+N) \\
\hat{\epsilon}_{\max } & =\arg \max \Phi(k)
\end{aligned}
$$


where $m$ is the correlation lag, $N_{\mathrm{CP}}$ is CP length in samples. Accordingly, the fractional part of the CFO is estimated from

$$
\hat{\nu}_{0}=-\frac{1}{2 \pi} \arg \left\{\Phi\left(\hat{\epsilon}_{\max }\right)\right\}
$$

The estimated $\hat{\theta}_{\max }$ could be used as the start position of the FFT window for further data demodulation processing. In reality, the estimated peak in $\Phi(k)$ may be strongly affected by multipath. To make sure that the start of the FFT window located in the CP, it is then suggested to assign the beginning of the FFT window to be preadvanced by $\lambda$ sample interval before the peak $\hat{\epsilon}_{\max }$, i.e.

$$
\hat{\epsilon}_{0}=\hat{\epsilon}_{\max }-\lambda
$$

Based on the coarse synchronization estimation $\left\{\hat{\theta}_{0}, \hat{\nu}_{0}\right\}$, the time and frequency offset is directly corrected on the received samples. The scatter pilot sequence detection can be started by removing the cyclic prefix and analyzing the signal in frequency domain. For this purpose, a FFT is applied to the samples belonging to a same OFDM symbol, which is expressed as

$$
d(n)=\mathcal{F}\left\{r\left(k+\hat{\epsilon}_{0}\right) e^{-j 2 \pi k \hat{\nu}_{0} / N}\right\} \cdot e^{j 2 \pi n \lambda_{0} / N}
$$

where $\mathcal{F}\{\cdot\}$ is the discrete-time transform operator.

2) Scattered pilot sequence detection: In DVB-T, the scatter pilot sequence is repeated in every 4 symbols. Therefore, to detect the scatter pilot sequence, after FFT, the sequence on the index of the possible scatter pilot subcarriers $p_{s}$ is cross-correlated with the sequence located 4 symbols later. By enumurating all the four possible index sequence in the set of the scattered pilot subcarriers $\mathcal{P}_{s}$, the maximum value among the four correlations gives the scattered sequence number of the current OFDM symbol.

$$
\begin{aligned}
R_{\mathrm{SPSD}}(m) & =\Sigma_{p_{s} \in \mathcal{P}_{s, m}} d_{i}\left(p_{s}\right) \cdot d_{i+4}^{*}\left(p_{s}\right), m \in\{0,1,2,3\} \\
\hat{m} & =\arg \max _{m}\left|R_{\mathrm{SPSD}}(m)\right|
\end{aligned}
$$

where $\mathcal{P}_{s, m}$ is the indexes of the $m$-th subset of scattered pilot subcarriers $\mathcal{P}_{s}$ and $\hat{m} \in$ $\{0,1,2,3\}$ denotes the estimated scattered sequence number.

3) Integer CFO Estimation: The estimated CFO obtained in the coarse symbol boundary detection has ambiguity in frequency [22]. In DVB-T, the continuous pilot subcarriers are unitized for integer $\mathrm{CFO}$ estimation. As the integer $\mathrm{CFO}$ causes frequency shift of the received 
frequency-domain signals, the correlation on a set of subcarriers of two consecutive symbols can be computed to detect the continuous pilot subcarriers [22]:

$$
R_{\mathrm{ICFO}}(g)=\Sigma_{p_{c} \in \mathcal{P}_{c}} d_{i}\left(p_{c}+g\right) \cdot d_{i+1}^{*}\left(p_{c}+g\right), g=0, \pm 1, \pm 2, \cdots
$$

where $\mathcal{P}_{c}$ denotes the set of continuous pilot indexes $p_{c}$. The integer $\mathrm{CFO}$ is found by searching for the maximum of this correlation function, i.e.,

$$
\hat{g}=\arg \max _{g}\left|R_{\mathrm{ICFO}}(g)\right|
$$

This estimator works well if the channel is considered as quasi-stationary, which will be the case in our test. Based on the previous operation, the integer CFO can be corrected by circularly shifting the subcarrier by $-\hat{g}$.

\section{B. Time delay acquisition}

By applying the above synchronization steps, the residual timing and frequency errors are small enough for the data demodulation. In terms of reliable data demodulation, such residual timing and frequency error is easily compensated by the channel equalization. It is therefore not necessary to have a very accurate timing synchronization to be able to demodulate the data message. However, for the purpose of positioning and navigation, further refinement steps that aim to find more accurate symbol timing are necessary. In this work, acquisition and tracking steps are further applied to achieve this end.

The acquisition step aims to reduce the residual errors resulting from the coarse synchronization and feed an accurate start point as the initial value for tracking. In the terrestrial transmissions such as DVB-T, the multipath effect is commonly encountered and sometimes is very severe. Therefore, we consider acquiring the multipath time delays and then detecting the first path of arrival. The acquisition method always requires very heavy computation if searching all the received signals in one OFDM symbol. In order to accelerate the method, we therefore start the step by truncating the research region.

1) the search region for acquisition: Considering the fact that multipaths arrive within a limited period of samples and based on the coarse synchronization results derived in Section III-A, which might be a biased estimation of the start of an OFDM symbol with a possibly large standard deviation [23], we set a acquisition region $D_{\rho}$, which is defined as $D_{\rho} \triangleq\left[\epsilon_{\max }-\frac{\rho}{2}, \epsilon_{\max }+\frac{\rho}{2}\right]$. The length of $\rho$ is required to include most power of the estimated 
channel delay, i.e.,

$$
S_{\rho} \geq \Gamma_{\rho} \sum_{k=0}^{N-1}\left|\hat{h}_{i, k}\right|^{2}
$$

where $\Gamma_{\rho}$ is a threshold value and $\hat{h}_{i, k}$ is the estimated channel impulse response (CIR) of the $i$ th symbol. The CIR $\hat{h}_{i, k}$ is estimated from the channel frequency response, which is further derived as follows.

Denote $c_{i}\left(p_{m}\right)$ as the local replica of the continuous and scatter pilots in the $i$ th symbol, where $p_{m} \in \mathcal{P}_{c} \cup \mathcal{P}_{s}$, and let $d_{i}\left(p_{m}\right)$ denote the received symbols of the pilot subcarriers. Then, the least square (LS) estimate of the channel frequency response at these pilot subcarrier frequencies are

$$
\hat{H}_{i}\left(p_{m}\right)=\frac{d_{i}\left(p_{m}\right)}{c_{i}\left(p_{m}\right)}
$$

We then apply a linear frequency interpolation from all the pilot subcarriers to $N_{u}$ full OFDM subcarriers and add zero padding for $N-N_{u}$ subcarriers, which are situated on the both edges to form the guard bands. Then a $N$ points IFFT is applied to obtain the estimation of the channel impulse response (CIR) $\hat{h}_{i, k}$. Empirically, by the pre-evaluations in our field tests, we set $\rho=100$. Within $D_{\rho}$, the $97 \%$ of the total channel power delay is included, which is robust enough to detect most of the multipaths with the following acquisition method.

2) multipath acquisition: We resort to the matching pursuit (MP) method [24] to acquire the multipath time delay in fractional sampling interval. The principle of MP is to utilize the sparseness of the CIR and aims to find the lowest dimensional linear combination of delayed versions of the transmitted symbol sequences to represent the received signal. Reference [6] applied a basic MP algorithm in time domain to find the multipath delays, which utilized the absolute value of the time-domain correlation function and decomposed it into a sum of absolute sinc functions, a correlation function between one signal replica and the local generated signal. The method is feasible to estimate the delay and absolute amplitude of the multipath components with a finer accuracy than the coarse synchronization algorithm, while the phase information of the channel coefficient is omitted in the estimate.

In this work, in order to simultaneously estimate the delays and complex channel coefficients, a frequency domain method is proposed for multipath acquisition, which utilizes the received scattered pilot subcarriers sequence $d_{i}\left(p_{s}\right)$ for signal decomposition. In addition, due to the unknown order of $\mathbf{h}$, the order-recursive least square MP (LS-MP) algorithm [25] is adapted to solve the problem of multipath acquisition. The method is described as follows. 
Taking advantage of the time shift property of the Fourier transform, i.e. $\mathcal{F}\{d(k \pm \tau)=$ $r(n) \cdot \exp \left( \pm j \frac{2 \pi n \tau}{N}\right)$, the time delay estimation is then equivalent to the problem of phase estimate in frequency domain. Therefore, the multipath acquisition problem here is to find $\hat{h}_{i, l}$ and $\hat{\tau}_{i, l}$ that minimize the error between the received scatter pilots and the local replica:

$$
\hat{h}_{i, l}, \hat{\tau}_{i, l}=\arg \min _{h_{i, l}, \tau_{i, l}} \sum_{p_{s} \in \mathcal{P}_{s}}\left\|d_{i}\left(p_{s}\right)-\sum_{l} h_{i, l} e^{j \frac{2 \pi p_{s} \tau_{l}}{N}} c_{i}\left(p_{s}\right)\right\|
$$

where $\hat{\tau}_{i, l}$ is within the searching region $D_{\rho}$, which is derived in III-B1. $l$ is the number of multipath, which is detected recursively.

Define the time delay sequence $\Upsilon=\left[0, \Delta \tau, \cdots,\left(N_{\tau}-1\right) \Delta \tau\right]+\hat{\epsilon}_{\max }-\frac{\rho}{2}$, where $\Delta \tau$ is time interval for the delay estimation and $N_{\tau}=\left\lfloor\frac{\rho}{\Delta \tau}\right\rfloor$. A matrix $\mathbf{C}$ is then constructed with the row element expressed as $\mathbf{c}\left(p_{s}\right)=c_{i}\left(p_{s}\right) \cdot \exp \left\{j \frac{2 \pi p_{s} \Upsilon}{N}\right\}$. The problem of (12) can be further converted to solve for $h$ from the following:

$$
\mathbf{d}=\mathbf{C} \cdot \mathbf{h}
$$

where $\mathbf{C}$ is a $N_{p} \times N_{\tau}$ matrix, $\mathbf{d}$ is $N_{p} \times 1$ vector with the element $d_{i}\left(p_{s}\right)$ and $\mathbf{h}$ is $N_{\tau} \times 1$ vector. A solution $\hat{\mathbf{h}}$ may be viewed as the coefficient vector associated with the representation of $\mathbf{d}$ in terms of the column of $\mathbf{C}$.

To apply the order-recursive LS-MP, we assume that after $q-1$ st iteration, the set of the identified columns is $\mathbf{C}_{s, q-1} \triangleq\left[\mathbf{c}_{s_{1}}, \mathbf{c}_{s_{2}}, \cdots, \mathbf{c}_{s_{q-1}}\right]$ and $I_{q-1} \triangleq\left\{s_{1}, s_{2}, \cdots, s_{q-1}\right\}$. Accordingly, the associated coefficient vector $\hat{\mathbf{h}}_{q-1}$ is obtained using the LS method, i.e.,

$$
\hat{\mathbf{h}}_{q-1}=\mathbf{R}_{q-1}^{-1} \mathbf{z}_{p-1}
$$

where

$$
\begin{aligned}
\mathbf{R}_{q-1} & =\mathbf{C}_{s, q-1}^{*} \mathbf{C}_{s, q-1} \\
\mathbf{z}_{q-1} & =\mathbf{C}_{s, q-1}^{*} \mathbf{d}
\end{aligned}
$$

The associated residual vector $\mathbf{r}_{q-1}$ can be calculated as

$$
\mathbf{r}_{q-1}=\mathbf{d}-\mathbf{C}_{s, q-1} \hat{\mathbf{h}}_{q-1}
$$

At the $q$ th iteration, the algorithm finds a new column out of the set of remaining columns, denoted by $\mathbf{c}_{s_{q}}$, where the column $s_{q}$ selected is according to the following optimization problem:

$$
s_{q}=\arg \max _{j \notin I_{q-1}}\left|\mathbf{c}_{j}^{*} \mathbf{h}_{q-1}\right|^{2}
$$


With the new column $\mathbf{c}_{s_{q}}$ selected, the matrix $\mathbf{R}_{q}$ and $\mathbf{C}_{q}$ are updated as

$$
\begin{aligned}
\mathbf{R}_{q} & =\left[\mathbf{C}_{s, q-1} \mathbf{c}_{s_{q}}\right]^{*}\left[\mathbf{C}_{s, q-1} \mathbf{c}_{s_{q}}\right] \\
\mathbf{z}_{q} & =\left[\mathbf{C}_{s, q-1} \mathbf{c}_{s_{q}}\right]^{*} \mathbf{d}
\end{aligned}
$$

The iterative search process stops when no more path satisfying $\left|h_{s_{q}}\right| \geq \Gamma_{\text {acq }}$ are detected in the searching region, where $\Gamma_{\text {acq }}$ is a threshold.

It has been shown in reference [25] that the selection of the column $\mathbf{c}_{s_{q}}$ in (17) gives the minimum squared residual error. Rather than picking the new column onto which the rank-one projection of the residual vector is maximized as the basic MP algorithm does, it seeks the new column which, together with all the previously selected columns, represents the received signal with the minimum squared residual error. As a result, the selected columns span a subspace onto which the projection of the received signal is maximized.

It is also noted that, the phase information derived from the complex-valued $h_{s_{q}}$ will be useful for the first path detection, especially when the receiver is static and the line-of-sight path exists. In such scenarios, the phase of the first path should be kept within small variance, which is affected only by the noise in the front-end of the receiver.

3) First path detection: This work is focused on the TOA estimation in static scenarios. To detect the first path of arrival, we apply the rule of the earliest and the most frequently detected path as the first path. In more details,

- for the successive $N_{\text {symbol }}$ OFDM symbols, get all the multipath delays acquired from the order-recursive LS-MP method, together with the phase information.

- get the occurrence probability of the detected multipath delays in $N_{\text {symbol }}$ OFDM symbols

- among all the most frequently detected paths (if any), choose the earliest path of arrival as the first path

- check the standard deviation of the phase of the first path of arrival is smaller than the threshold $\varphi_{\mathrm{TH}}$ in $N_{\text {symbol }}$ OFDM symbol time.

\section{Delay tracking}

Once the first path is acquired, expressed in fractional sample time, tracking loops are then implemented to filter the time delay and to achieve even more accurate TOA estimation. Similar as the multipath acquisition, the time delay is also implemented in frequency domain by utilizing the time shift property of Fourier transform. The detailed operation of the DLL is described as follows. With the results estimated from the multipath acquisition, the time 
delay of the first path has been estimated with fractional sample accuracy. Therefore, the receiver timing is first adjusted by using the estimated normalized symbol timing error. We can write the phase adjusted received pilots as

$$
\hat{d}_{i}(p)=e^{-j \frac{2 \pi p \hat{\tau}}{N}} d_{i}(p)
$$

where $\hat{\tau}$ is the estimated normalized symbol delay. The received pilots are then crosscorrelated with the locally generated early and late reference pilots. The locally generated early and late reference pilots are given by

$$
\begin{gathered}
c_{i, e}(p)=e^{+j \frac{2 \pi p \xi}{N}} c_{i}(p) \\
c_{i, l}(p)=e^{-j \frac{2 \pi p \xi}{N}} c_{i}(p)
\end{gathered}
$$

where $\xi(0<\xi<1 / 2)$ is the advanced (and retarded) interval which is normalized to the OFDM sample interval. Let us define a modified cross-correlation function of the received scatter pilots with its replica as

$$
R_{i}(\epsilon)=\frac{1}{N_{p}} \sum_{p \in \mathcal{P}_{s}} \hat{d}_{i}(p) \cdot c_{i}^{*}(p)
$$

where $\epsilon=\tau-\hat{\tau}, N_{p}$ is the number of the scatter pilot subcarriers in $i$ th OFDM symbol. Accordingly, we can write the early cross-correlation branch output as

$$
\begin{aligned}
R_{i, e}(\epsilon) & =\frac{1}{N_{p}} \sum_{p \in \mathcal{P}_{s}} \hat{d}_{i}(p) \cdot c_{i, e}^{*}(p) \\
R_{i, l}(\epsilon) & =\frac{1}{N_{p}} \sum_{p \in \mathcal{P}_{s}} \hat{d}_{i}(p) \cdot c_{i, l}^{*}(p)
\end{aligned}
$$

We apply the Early-Minus-Late Power (EMLP) discriminator for DLL tracking. The normalized discriminator is expressed as:

$$
a_{i}(\epsilon)=\frac{1}{k_{d}}\left(\left|R_{i, e}(\epsilon)\right|^{2}-\left|R_{i, l}(\epsilon)\right|^{2}\right)
$$

where the normalization factor $k_{d}$ is to keep $a_{i}(\epsilon) \approx \epsilon$ when $\epsilon \rightarrow 0$. By smoothing the discriminator $a_{i}(\epsilon)$ with a loop filter, the delay estimate of the $(i+1)$ th OFDM symbol is updated by

$$
\hat{\tau}_{i+1}=\hat{\tau}_{i}+\tilde{\epsilon}_{i}
$$

where $\tilde{\epsilon}_{i}$ is the output from the loop filter of the $i$ th symbol. 


\section{Performance Analysis on tOA estimation}

\section{A. Expression of ideal autocorrelation function (ACF)}

We start the performance analysis from the ideal autocorrelation function of the pilots. Without the noise, the normalized ideal ACF of pilot sequence is calculated as

$$
\begin{aligned}
R_{i}^{o}(\epsilon) & =\frac{1}{N_{p}} \sum_{p \in \mathcal{P}_{s}} c_{i}(p) \cdot\left(c_{i}(p) \cdot e^{-j \frac{2 \pi p \epsilon}{N}}\right)^{*} \\
& =\frac{1}{N_{p}} \sum_{p \in \mathcal{P}_{s}} c_{i}(p) \cdot c_{i}^{*}(p) \cdot e^{j \frac{2 \pi p \epsilon}{N}}
\end{aligned}
$$

In DVB-T, the pilots are inserted every 12 subcarriers. Denote $\mathrm{E}\left[c_{i}(p) \cdot c_{i}^{*}(p)\right]=A$, where $\mathrm{E}[\cdot]$ denotes statistical expectation. Thus (25) is equal to

$$
R_{i}^{o}(\epsilon)=\frac{1}{N_{p}} A e^{\frac{j \pi\left[2 p_{i}(0)+12\left(N_{p}-1\right)\right] \tau}{N}} \frac{\sin \pi 12 N_{p} \epsilon / N}{\sin \pi 12 \epsilon / N}
$$

where $p_{i}(0)$ is the index of the first scatter pilot in $i$ th symbol. Furthermore, since $N>>1$ (for a commonly used $8 \mathrm{~K}$ mode in DVB-T, $N=8196$ ) and for small values of $\tau$ within several samples, the following approximations validate: $\left[2 p(0)+12\left(N_{p}-1\right)\right] / N \approx 1$ and $\sin \frac{\pi 12 \epsilon}{N} \approx \frac{\pi 12 \epsilon}{N} \rightarrow 0$. Therefore, the correlation function (26) can be simplified as

$$
R_{i}^{o}(\epsilon)=A e^{j \pi \epsilon} \operatorname{sinc}(\pi \beta \epsilon)
$$

where $\beta=\frac{12 N_{s p}}{N} \approx 0.832$.

The ideal ACF is shown in Figure 3. As a comparison, the ACF of GPS C/A code and the Galileo E1 code are also presented. From Figure 3, due to the wider bandwidth the DVB$\mathrm{T}$ signals occupy, the mainlobe in the ACF of DVB-T signal is much narrower (about $\frac{1}{8}$ ) than for GPS L1 C/A Galileo E1, which suggests the increased possibility of detecting two multipaths, especially when their arrival time is larger than one chip of the DVB-T signal (about $0.11 \mu \mathrm{s}$ ). Meanwhile, by observing the peak of three ACFs in Figure 3, the peak of the DVB-T signal is smoother than the other two, which suggests the difficulty for DVB-T signals to differentiate two closely spaced multipaths arrived less than $0.11 \mu$ s. However, it should be noted that the ACF only takes into account ideal GNSS signals, which implies an infinite bandwidth for GNSS receivers. However, in reality, the front-ends of the GNSS receiver have a limited RF bandwidth, as low as $2 \mathrm{MHz}$ for low-cost receivers, which will smooth the peak of the ACF. Nevertheless, this is not true for DVB-T receivers, because DVB-T signals are always applied with a strictly limited bandwidth. 


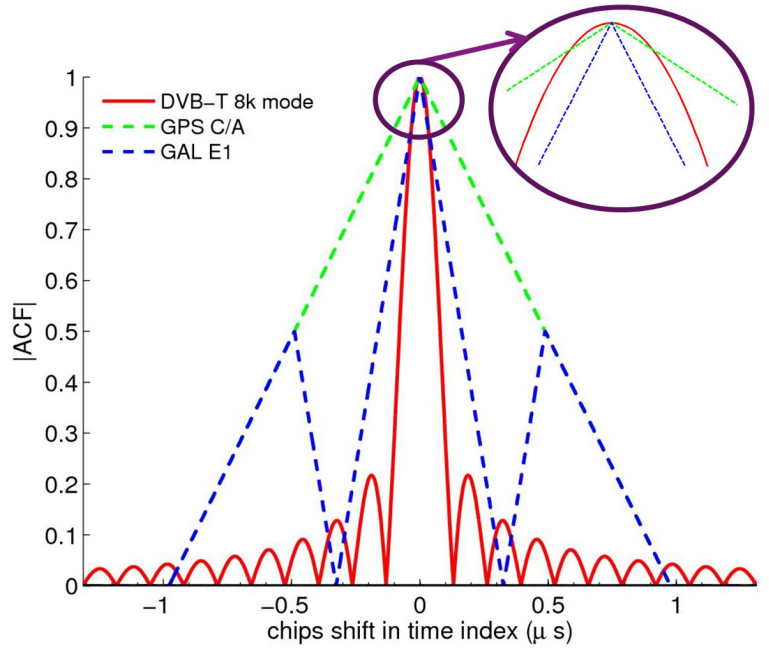

Fig. 3. Ideal ACF of DVB-T, GPS C/A and GALILEO E1

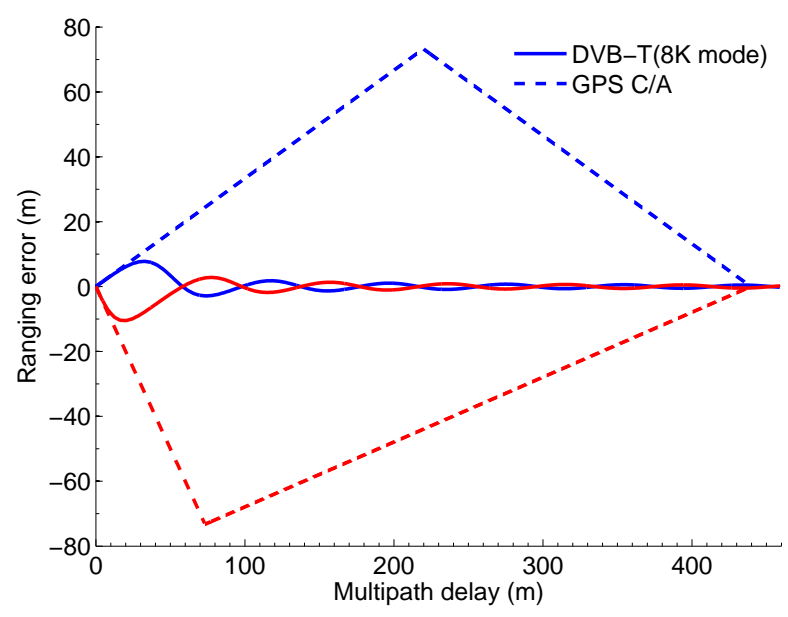

Fig. 4. Multipath error envelope of EML for DVB-T and GPS C/A, where $\xi=1 / 2$

\section{B. The influence of multipath on delay tracking}

In GNSS, multipath has an important impact on the tracking error in the DLL based receiver [26]. To assess the DLL sensitivity to multipath, the multipath error envelop (MEE) of DVB-T and GPS C/A signals are shown in Figure 4, where the maximum negative and positive errors on delay estimation is computed by setting the delay of one multipath of amplitude equal to half of the amplitude of the LOS signal. From the MEE in Figure 4, it is observed that the ranging errors of DVB-T ( $8 \mathrm{~K}$ mode) are much smaller than the GPS C/A signal, which is coherent with the discussions of the ideal ACF shown in Figure 3. 


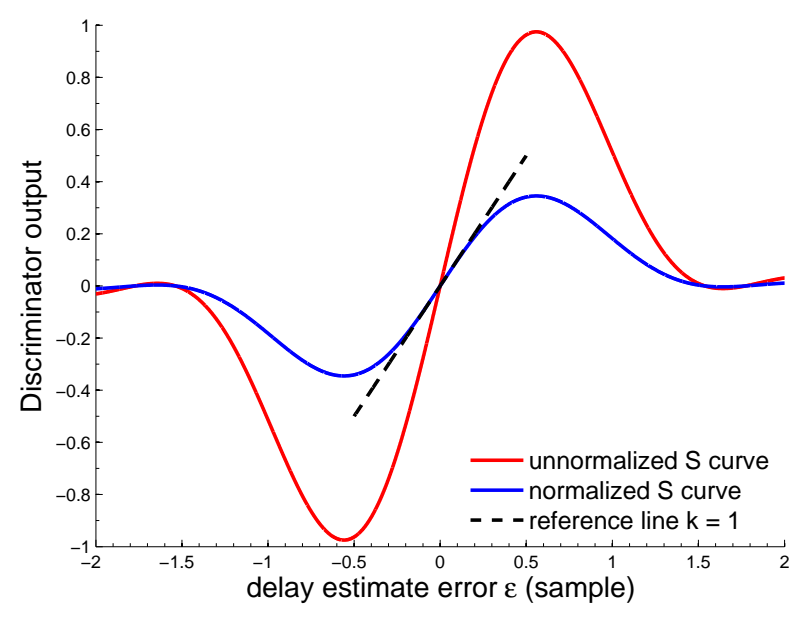

Fig. 5. DLL S curve $(\xi=1 / 2)$

\section{C. $S$ curve of the EMLP discriminator}

Considering an AWGN (additive white Gaussian noise) channel with a high enough SNR, the (un)normalized S-curve $S(\epsilon, \xi)$, which is the tracking errors from the EMLP discriminator, is illustrated in Figure 5, where $\xi=1 / 2$. The whole derivation is shown in Appendix A.

\section{TEST BENCH FOR DVB-T SIGNAL SAMPLING}

In this work, a USRP [27] based test platform is built for signal sampling and recording. USRP is an Ettus Research LLC [27] product, which is a low-cost, flexible and opensource device for making software-defined radio. A USRP is internally composed by a motherboard and a daughterboard. The USRP motherboard is responsible for clock generation and synchronization, digital-analog signals interfacing, host processor interfacing, and power management, while the USRP daughterboard is used for up/down-conversion, analog filtering, and other analog signal conditioning operations.

In the presented test bench, we use USRP N210, which has integrated a motherboard including a Xilinx Spartan 3A-DSP 3400 FPGA, 100 Msps dual analog-to-digital converter (ADC), 400 Msps dual DAC, and GE connection with the host PC. We adopt the WBX daughterboard, which allows to receive signal between $50 \mathrm{MHz}$ and $2.2 \mathrm{GHz}$ with a bandwidth up to $40 \mathrm{MHz}$. It also proposes an amplifier with an adjustable gain from 0 to $31.5 \mathrm{~dB}$, with a noise figure between 5 and $7 \mathrm{~dB}$. Such configuration is able to be well adapted to the French DVB-T signal bandwidth and carrier frequency (see Table I). 


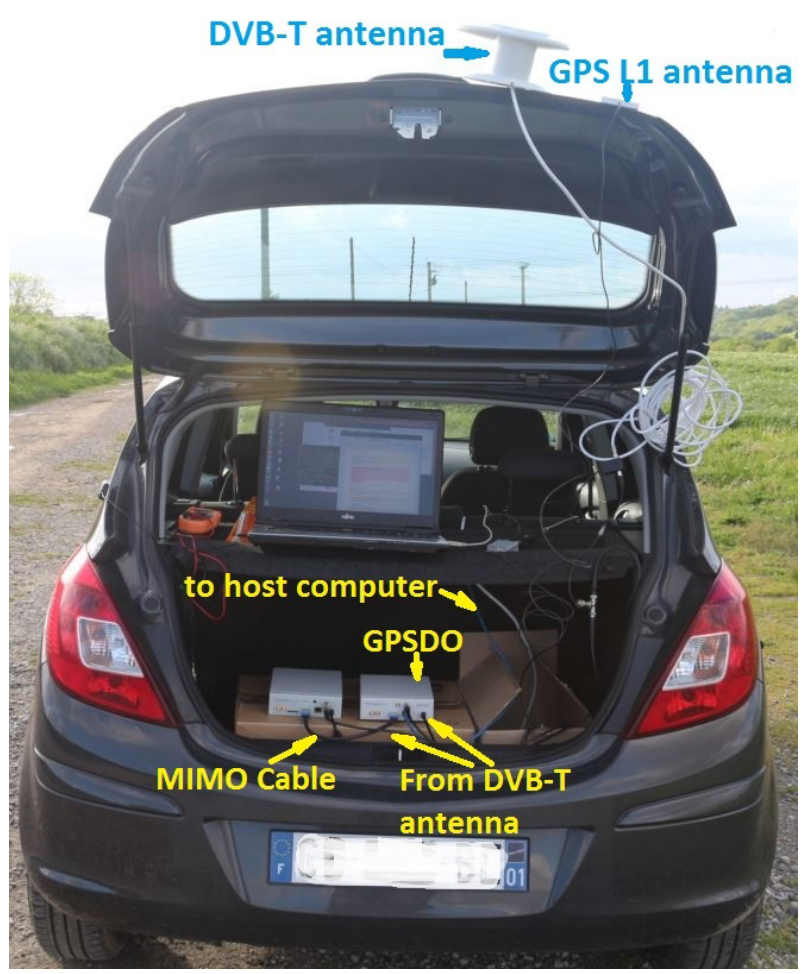

Fig. 6. USRP based test bench for outdoor testing

To allow the local sampling clock synchronized to GPS time, a GPSDO (GPS Disciplined Oscillator) module [28] is inserted in USRP N210, which is to provide $10 \mathrm{MHz}$ reference input and allows the master oscillator of the USRP2 locking on an external clock. A 1 pulse per second (1PPS) input allows absolute timing operations. According to the specification, the accuracy of the 1 PPS of the GPSDO is within \pm 50 ns to UTC RMS (1-Sigma) when the GPS is locked. The GPSDO is also able to output the GPS position, with an approximate accuracy of only 15 meters. Additionally, for the success of the outdoor field tests, another USRP is used as a backup equipment to sample the signals simultaneously. A MIMO cable is used to connect these two USRPs.

There are two antennas used in the test platform. One is the DVB-T antenna, which is a mass-market amplified mobile omnidirectional UHF antenna ELAP 240011-40 dB gain, 1.1 $\mathrm{dB}$ noise factor (vendor information). The other one is the GPS L1 antenna, which provides the GPS signals to GPSDO module to generate a synchronized sampling clock. Figure 6 shows the USRP based test bench for the outdoor testing. 


\section{OUTDOOR EXPERIMENTS AND RESULTS}

To quantify the performance of the TOA tracking on real signals, field tests were carried out in five cities in Southern France. Table II and Table III summarize all the testing scenarios. The signals are sampled by the USRP frontend described in Section V. During the tests, the receivers are kept static in open area of outdoor fields and the localization of the receiver is obtained from the GPSDO by inquiring a specific command. The TOA tracking results are shown and discussed in Section VI-A. With the tracking results, it is also possible to infer whether or not the clock of the emitters are synchronized with GPS time, which is crucial for TOA based positioning. Section VI-B presents the tracking results where the emitter is found to have a clock drifting with reference to GPS time. Moreover, from the sampled signals, multiple emitters within SFN are also observed during the tests. Section VI-C describes the acquisition results from multiple emitters in different cities. The distance difference between different emitters and the receiver is calculated and their inconsistence with the geometric distance difference is found and discussed. Such inconsistence will be taken into account for the future TDOA based positioning with DVB-T signals.

\section{A. TOA tracking results}

Two tests are presented in more details, within which one test shows the tracking results with relatively high $C / N_{0}$ signals received, while the other shows the performance on weak signals. All the tracking results in the outdoor testing campaigns are summarized and presented thereafter. The estimation of $C / N_{0}$ is referred to the method used in the GNSS field [29, pp. 391-392]. In this work, we set $M=20$ and $K=5$ for 0.1 s average. In all the following tests, to get the multipath acquisition, we set $\Delta \tau=0.5$. In the DLL loop, the bandwidth is set as $100 \mathrm{~Hz}$ to ensure the loop convergence and only observe the emitter clock behavior.

1) Marseille Testing Scenario: In the test of Marseille, the signal from the emitter of "Grande Etoile" is tracked. The height of the antenna is about $40 \mathrm{~m}$. The signals are transmitted from $10 \mathrm{~kW}$ to $100 \mathrm{~kW}$ for different channels. In the test shown as follows, the carrier frequency of the signal is in Channel 30, which is $546.166667 \mathrm{MHz}$ and the signals are emitted with an ERP (Effective Radiated Power) of $100 \mathrm{~kW}$. It is noted that, the fractional part of the carrier frequency, i.e. $0.166667 \mathrm{MHz}$ is a spectrum protection offset [30]. The receiver is located in a parking place near the Notre Dame de la Garde, which has a 


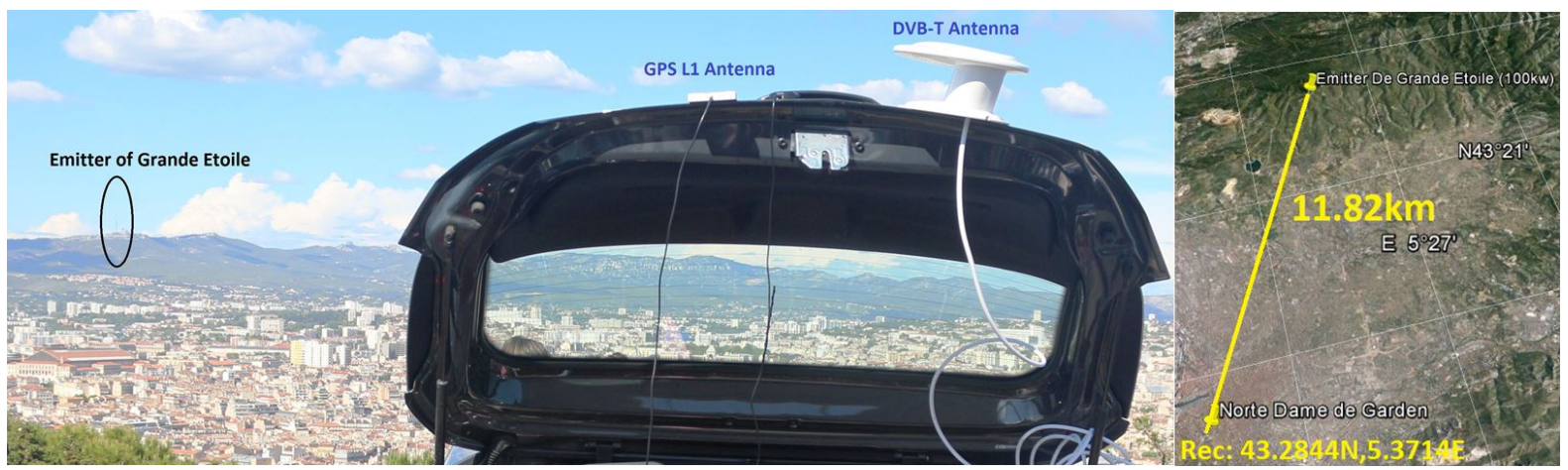

Fig. 7. Testing Scenario in Marseilles

direct sight view to the emitter. The distance between the emitter of "Grande Etoile" and the receiver is $11.8 \mathrm{~km}$. The testing scenario is shown in Figure 7.

The spectrum of an ideal DVB-T signal with $8 \mathrm{~K}$ mode $8 \mathrm{MHz}$ (effective) bandwidth and the sampled signals are shown in Figure 8, where the null margins to avoid the out-ofband emissions and pilot subcarriers that have more power than the payload data are clearly presented. Figure 9 shows the coarse synchronization of the DVB-T signals, which is averaged over 4 OFDM symbols. The maximum value of the auto-correlation $\hat{\epsilon}_{\max }$ is located at 1564 in sample and the corresponding fractional CFO $\hat{\nu}_{0}$ is estimated to $0.00012 \mathrm{rad} / \mathrm{s}$. After the FFT operation and the scatter pilot sequence detection, the estimated integer CFO $\hat{g}=0$. Figure 10 shows one snap shot of the multipath acquisition, where the threshold is set as the $80 \%$ of the total power within the acquisition region $D_{\rho}$. As shown in Figure 10, due to the low threshold value, it is possible that some of the detected paths are only the random noise in the receiver. By further applying the statistic method for all the acquired paths, the first path is detected, which is shown in Figure 11. From Figure 11, it is observed that the path arriving at $1564.5,1565.5$ and 1566.5 in sample are the three most frequently detected paths with the occurrence probability equal to 1 . Thus, among the three, the earliest path arriving at 1564.5 in sample is detected as the first arrived path. It is also observed that the phase standard deviation of the detected first path of arrival is smaller than the $\varphi_{\mathrm{TH}}$, where $\varphi_{\mathrm{TH}}$ is empirically set equal to 1 . This path is also set as the initial value for the DLL tracking. Figure 12 shows the 20-second tracking results from Marseille. From the tracking results, the $95 \%$ accuracy is within 0.95 meters, and the estimated $C / N_{0}$ is $57.97 \mathrm{~dB}-\mathrm{Hz}$. It is also noticed that there is a small slow-varying fluctuation from the output of the tracking results, which enlarges the $\pm 2 \sigma$ tracking error slightly. The fluctuation might be caused by the clock 

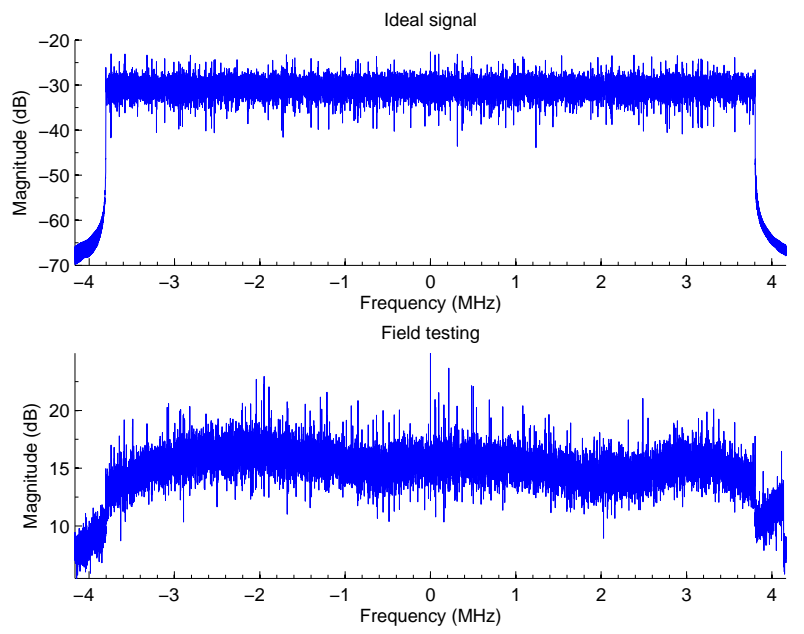

Fig. 8. Spectrum of ideal and field testing DVB-T signals (8K mode $8 \mathrm{M}$ bandwidth)
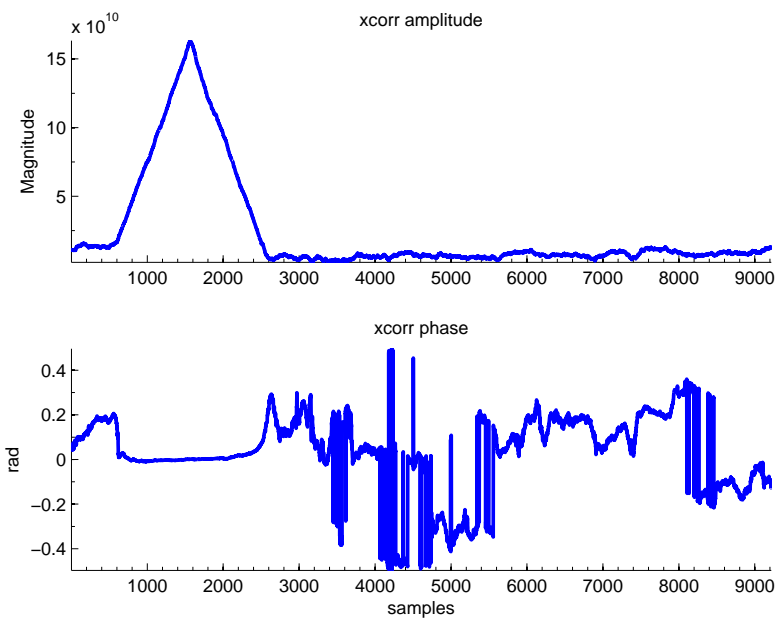

Fig. 9. Coarse synchronization results in Marseille test

drift of the transceiver's clock.

2) Toulouse, ENAC, Signav roof Scenario: In the test of Toulouse, the DVB-T antenna is mounted on the roof of the ENAC Signav lab and the test bench is synchronized with signals from the GPS antenna. The signal sampled for TOA estimation is emitted from "Pic de Nore", which is a regional emitter dedicated for broadcasting to Carcassone area. The emitter has an antenna height of $89 \mathrm{~m}$, an ERP of $8 \mathrm{~kW}$, and the carrier frequency of the signal is Channel 31, which is 554. $166667 \mathrm{MHz}$. The distance between ENAC Signav roof atenna to "Pic de Nore" emitter is $80.78 \mathrm{~km}$. The testing scenario is shown in Figure 13.

Figure 14 shows the results of channel acquisition, where 5 paths are detected, composed 


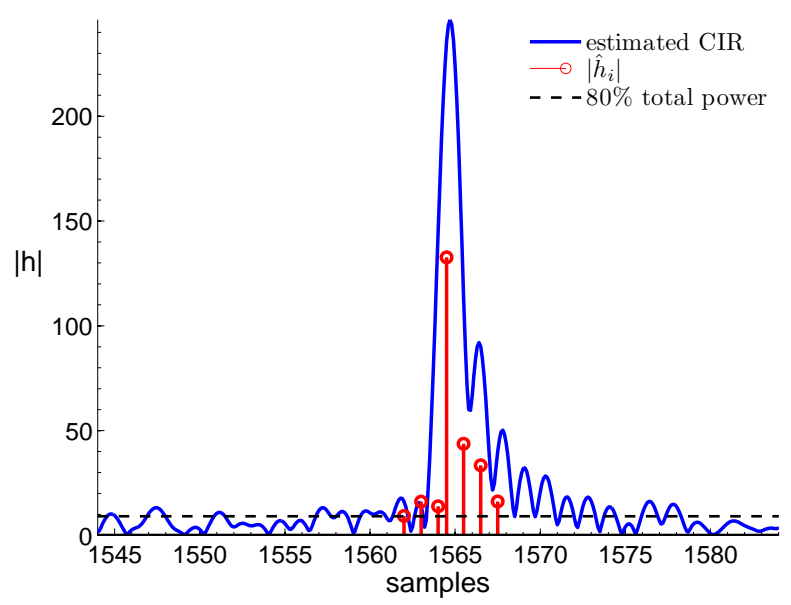

Fig. 10. Channel acquisition results in Marseille test
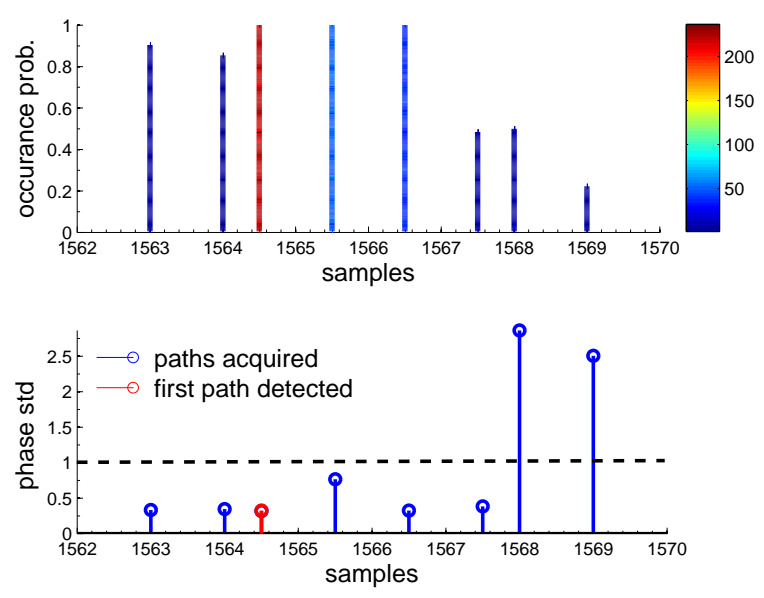

Fig. 11. The first path detection in Marseille test

of $80 \%$ power of the whole CIR. With the first path detected as 2960.5 in sample and put into tracking loop, the tracking results are shown in Figure 15, where the $95 \%$ confidence (2 sigma) interval is 3.1818 meters (0.095 samples) and the estimated $C / N_{0}$ is $49.56 \mathrm{~dB}-\mathrm{Hz}$. The fluctuation is also observed from the 45-second output of the DLL, which suggests the existence of slight errors of the clock between the emitter and the receiver.

3) summary of all the static tests: Table II summarized all the testing scenarios in five cities of the Southern France, which includes the location of the receivers, the parameters of the emitters and the distance between the transceivers. Figure 16 plots all the tracking errors (95\% accuracy) versus the $C / N_{0}$ estimated.

From the test results shown above, it is clear that the SDR DVB-T receiver is able to 


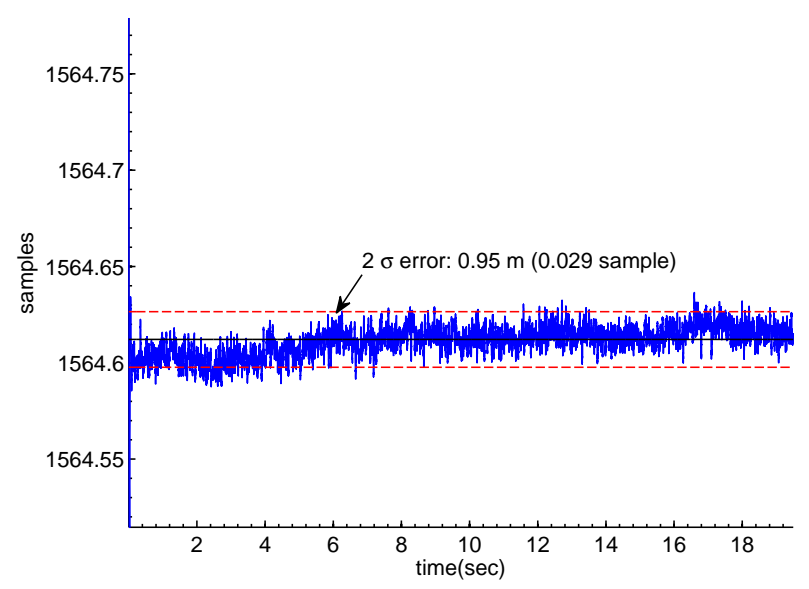

Fig. 12. TOA tracking results in Marseille test, where the estimated $C / N_{0}$ is $57.97 \mathrm{~dB}-\mathrm{Hz}$

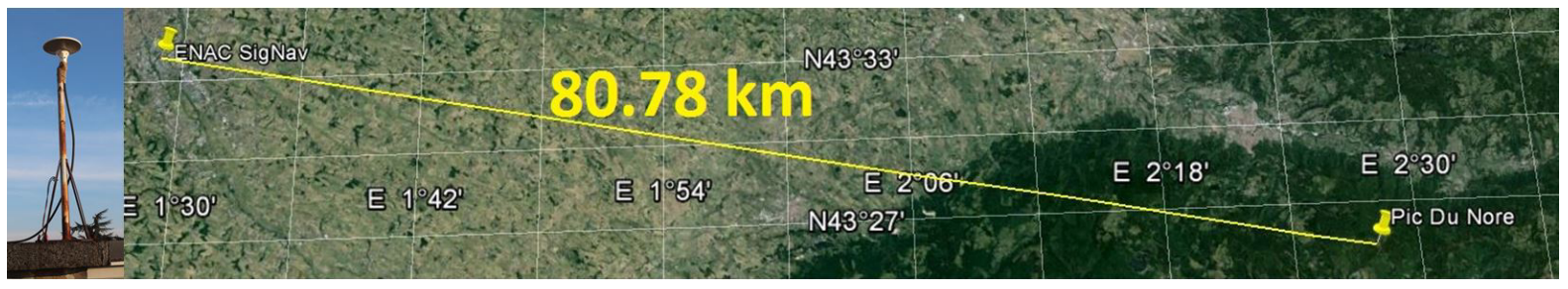

Fig. 13. Tests from ENAC roof antenna to the emitter of Pic de Nore

process effectively the raw data sampled from the USRP to get the TOA tracking estimation. All the tracking errors are within 4 meters (95\% accuracy), even for the signals received from the emitter of the adjacent city (e.g. in Toulouse test, the Test 1 in Table II), which is over $80 \mathrm{~km}$ away. The test results suggest a tendency that the signals with higher $C / N_{0}$ has smaller tracking errors, which is consistence with the theoretical analysis. Meanwhile, there are small jitters in the output of the delay tracking loop (see Figure 12 and Figure 15), which implies that the clocks of the transceiver are not perfectly synchronized. This is reasonable since both of the clocks are only designed to be synchronized to GPS signals. Due to the limitation of the physical equipment, some endurance of the errors for the synchronization is allowed. As an example, in the GPSDO module inserted in the USRP to provide the sample clock for the receiver, the error is acclaimed within $50 \mathrm{~ns}$ accuracy [28]. As an uncontrollable random variable, the non-perfectly synchronized clocks enlarge the tracking errors during the tracking process. 


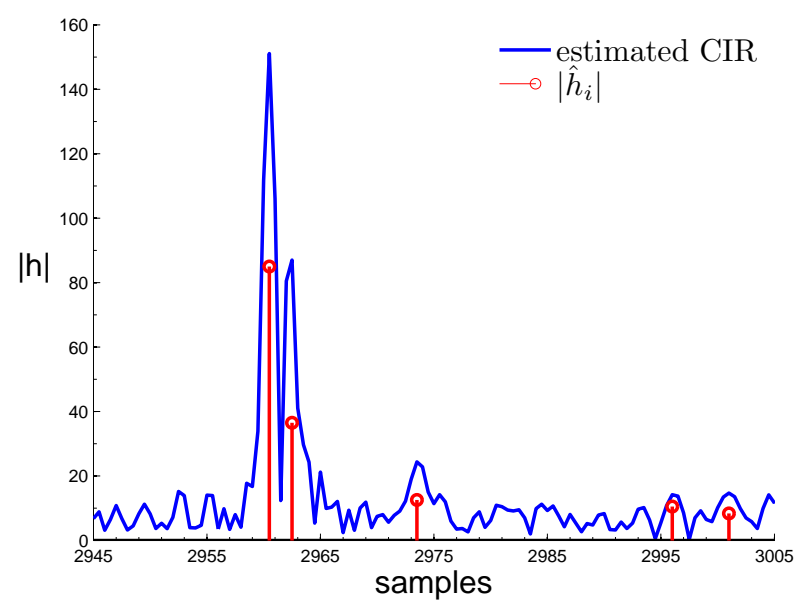

Fig. 14. Acquisition results from ENAC roof antenna to the emitter of Pic de Nore

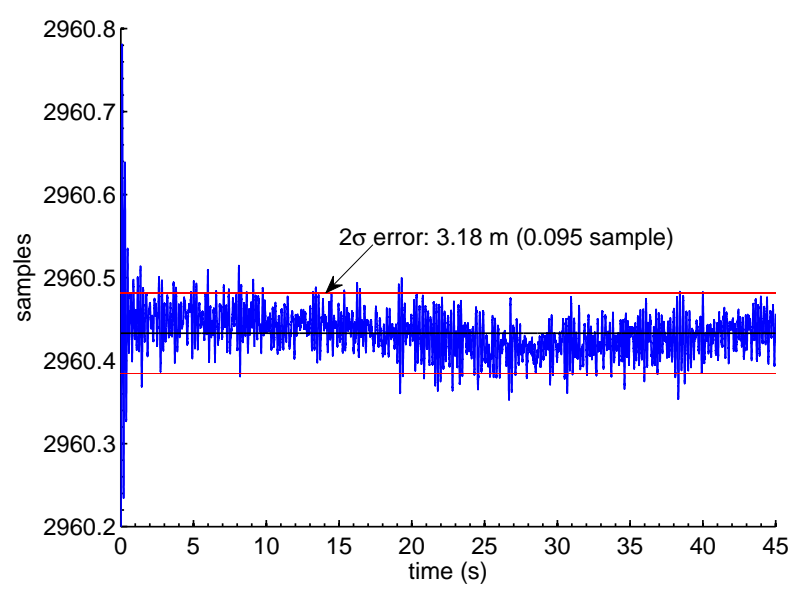

Fig. 15. TOA tracking results of signals from SigNav lab roof antenna in Toulouse. The interval of $2 \sigma$ error is $3.1818 \mathrm{~m}$ (0.095 sample) with the $C / N_{0}$ estimated as $49.56 \mathrm{~dB}-\mathrm{Hz}$.

B. clock drift testing of DVB-T emitters (T6)

It is in the ETSI standard that the emitter is suggested to be synchronized to GPS time. Based on the TOA tracking results, it is also possible to test such clock synchronization of the emitter. Due to the synchronized GPSDO within the USRP receiver, it has been observed that the emitters tested in Test 1 to Test 5 in Table II are synchronized with the GPS clock, since only small jitters are observed in the receiver during the whole tracking process for 20 to 45 seconds. The reason has already been discussed in Section VI-A. However, in a test to the Toulouse local emitter (T6), a large drift is observed. During the test, the signal is 


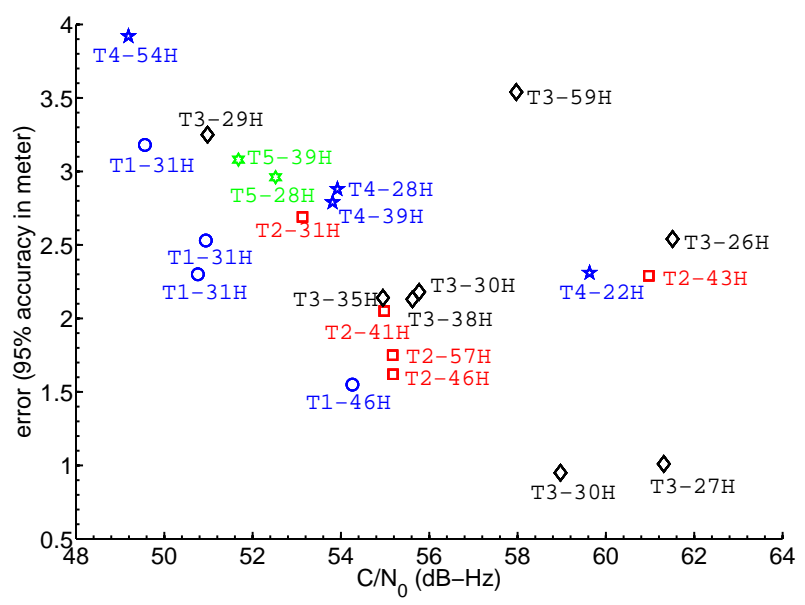

Fig. 16. Summary of all the TOA tracking results

TABLE II

STATIC TESTING SCENARIOS

\begin{tabular}{|c|c|c|c|c|c|c|}
\hline \multirow[b]{2}{*}{ Test } & \multicolumn{2}{|c|}{ Receiver } & \multicolumn{3}{|c|}{ Emitter [30] } & \multirow{2}{*}{$\begin{array}{c}\text { Distance } \\
\text { Tx \& Rx (km) }\end{array}$} \\
\hline & zone & coordinate & zone & cite & power $(\mathrm{kW})$ & \\
\hline $\mathrm{T} 1$ & Toulouse & $43.5614 \mathrm{~N} 1.4815 \mathrm{E}$ & Carcassone & Pic de Nore & 8 & 80.78 \\
\hline $\mathrm{T} 2$ & Carcassonne & $43.2026 \mathrm{~N} 2.3725 \mathrm{E}$ & Carcassonne & Pic de Nore & 8 & 25.83 \\
\hline $\mathrm{T} 3$ & Marseille & 43.2848 N 5.3717 E & Marseille & Grande Etolie & $10-100$ & 11.82 \\
\hline $\mathrm{T} 4$ & Nice & 43.7266 N 7.3497 E & Saint-Raphael & Pic de L'Ours & 63 & 45.37 \\
\hline $\mathrm{T} 5$ & Cannes & $43.5471 \mathrm{~N} 7.0130 \mathrm{E}$ & Saint-Raphael & Pic de L'Ours & 63 & 11.76 \\
\hline T6 & Toulouse & $43.5614 \mathrm{~N} 1.4815 \mathrm{E}$ & Toulouse & Lafilair & 2 & 4.26 \\
\hline
\end{tabular}

received from the roof antenna on the SigNav lab in ENAC, Toulouse. The carrier frequency of the sampled signal is channel $40(594.16667 \mathrm{MHz})$. The distance between the emitter and the receiver is $4.2 \mathrm{~km}$. Figure 17 shows the tracking results of the test, where the $95 \%$ confidence ( 2 sigma) interval is 21.90 meters within the tracking time of $20 \mathrm{~s}$. It is obvious that the drifted errors are out of the range of the accuracy for a feasible clock aligned with GPS signal. The results suggest that this emitter is not synchronized to GPS time.

\section{TOA from multiple emitters}

Tests were carried out to observe the multiple emitters in SFNs. Figure 18 demonstrates the acquisition results sampled from the roof antenna on the Signav lab of ENAC. Two peaks are found from the signal acquisition, one is from the Toulouse local emitter and the other is from the emitter of the Pic di Midi. The carrier frequency of the sampled signal is channel 


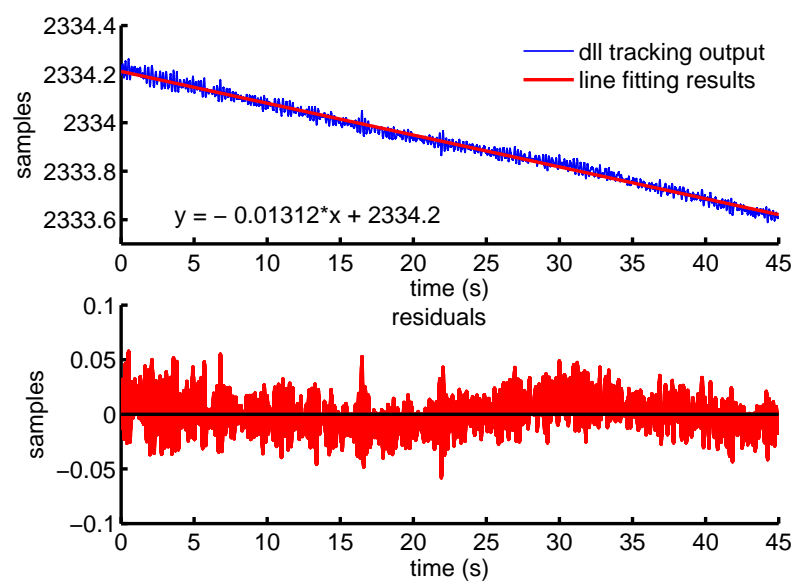

Fig. 17. TOA tracking results of signals from Toulouse local emitter

$40(594.16667 \mathrm{MHz})$.

Having multiple emitters in SFN is an advantage for time difference of arrival (TDOA) based positioning with DVB-T signals. However, by measurements, the time difference of the two peaks is 420.5 sample, which is equivalent to $13.78 \mathrm{~km}$ in distance. By comparison, the geometric distance difference of the two emitters to the receiver is $77.8 \mathrm{~km}$. Therefore, the distance difference is inconsistent between the field test and the geometric calculation. One factor that causes such inconsistency might be the intentional delay of the emission from the emitters in the SFN. In DVB-T system, a special packet called a megaframe initialization packet (MIP) inserted in the MPEG-2 TS (Transmission Streaming) controls the the emission time of the next mega-frame [31]. In the test, since the geometry distance difference has exceeded the CP length of the OFDM signal, which is $33.587 \mathrm{~km}$ in distance, the intentional delay is introduced so that the self-interference by the signals from the two SFN emitters only occurs in the CP length, which is much easier to be mitigated by channel equalization. Another factor that affect the inconsistency might be the lack of the line-of-sight signal to the receiver from the emitters.

Such inconsistency is also observed in the tests of other cities. Table III summarizes all the tests for the multiple emitters, where the location of the receiver in the same as the tests in Table II and the inconsistency of the distance difference is numerated.

It is noted that the problem of the inconsistency should be investigated in more details for the application of TDOA based positioning. However, such topic is beyond the scope of this paper, and thus, will be left for the future research. 


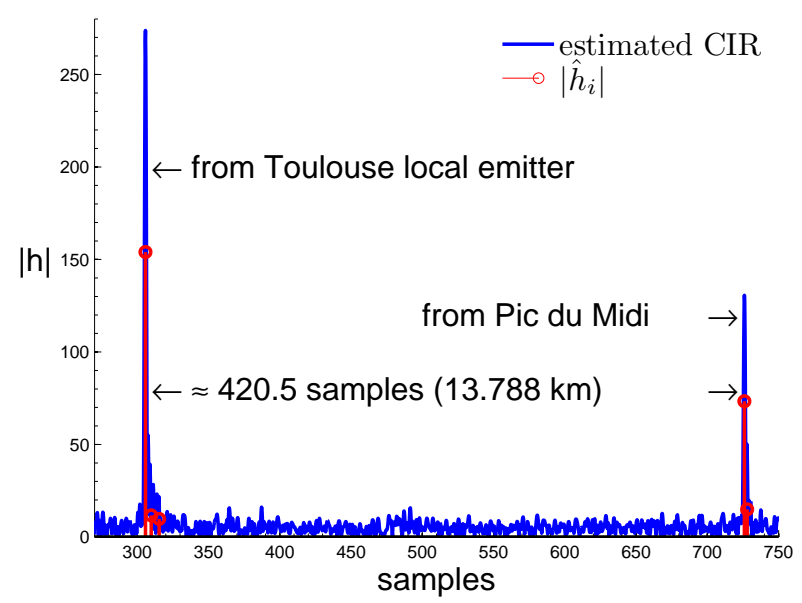

Fig. 18. TOA acquisition results from Pic di Midi and Toulouse local emitter

TABLE III

STATIC TESTINGS ON MULTIPLE EMITTERS

\begin{tabular}{|c|c|c|c|c|c|c|}
\hline \multirow[b]{2}{*}{ Test } & \multirow{2}{*}{$\begin{array}{c}\text { Receiver } \\
\text { zone }\end{array}$} & \multicolumn{3}{|c|}{ Emitter [30] } & \multicolumn{2}{|c|}{ Distance difference $(\mathrm{km})$} \\
\hline & & zone & cite & power $(\mathrm{kW})$ & geometry [30] & TOA acq. \\
\hline \multirow{2}{*}{ T6 } & \multirow{2}{*}{ Toulouse } & Toulouse & Pic du Midi & 13 & \multirow{2}{*}{77.8} & \multirow{2}{*}{13.8} \\
\hline & & Toulouse & Lafilair & 2 & & \\
\hline \multirow{2}{*}{$\mathrm{T} 7$} & \multirow{2}{*}{ Carcassonne } & Carcassonne & Pic de Nore & 8 & \multirow{2}{*}{22.8} & \multirow{2}{*}{15.8} \\
\hline & & Carcassonne & Carcassonne 2-sub & 0.010 & & \\
\hline \multirow{2}{*}{$\mathrm{T} 8$} & \multirow{2}{*}{ Marseille } & Marseille & Grande Etolie & $10-100$ & \multirow{2}{*}{6.5} & \multirow{2}{*}{11.3} \\
\hline & & Marseille & Pomegues & 3.5 & & \\
\hline \multirow{2}{*}{ T9 } & \multirow{2}{*}{ Cannes } & Saint-Raphael & Pic de L'Ours & 63 & \multirow{2}{*}{6.8} & \multirow{2}{*}{12.6} \\
\hline & & Cannes & Vallauris & 0.085 & & \\
\hline
\end{tabular}

\section{The applicability of the method for positioning}

In terms of applying the presented method for positioning, at least 3 DVB-T emitters are required to form the triangulation solution. According to our field test campaign in Southern France, it is not very common that enough TV repeaters are available in one city and in the same frequency channel at the moment. In reality, the most common case is that only two emitters are setup for transmitting DVB-T signals in one city. However, as the test results presented in Section VII.A. 2), with the presented method, the receiver was capable to track the signals from an emitter of an adjacent city for TOA estimation. Therefore, if the total number of emitters from the local and the adjacent cities is no less than 3 , it would be possible to compute the position with triangulation by estimating the TOA of the DVB-T 
signals from emitters in a local city and the adjacent cities. It should be noted that such method to include the adjacent emitters for triangulation requires the receiver to be able to process TV signals from different frequency channels. Indeed, adjacent TV emitters may broadcast the TV signal on different frequencies in order to avoid interferences.

As another possible application of the method, the ranging estimation from DVB-T signal can also be easily combined with the pseudorange estimates from a GNSS receiver, which achieves a DVB-T and GNSS hybrid positioning. Considering the high accuracy of the TOA estimation of DVB-T signals, the hybrid positioning solution could improve the positioning accuracy compared to GNSS only results.

When considering the application of positioning for use in practice, it is suggested that more information of the emitters should be provided as a prior information to the user, including the status of the emitters clock (i.e. synchronized or unsynchronized to GPS), the precise geolocation of the emitter (latitude, longitude, height), and the precise transmission time of the signals (normalized to the samples in one symbol). Such information would be necessary to implement a reliable DTV-based positioning service. These information could be inserted in the blank segment of the signal format that has not been used ever or in the data server that can be easily accessed for the users. Note that this kind of information does not vary in time, so it does not need to be transmitted with a high repetition rate.

\section{CONCLUSIONS}

In this paper, the problem of TOA estimation of DVB-T signals for the application of positioning is studied both from theoretical analysis and the practical field testing point of views.

Theoretical analysis showed that, compared to GNSS signals, the DVB-T signals with narrower main lobe of the autocorrelation and smaller multipath error envelop are promising to be utilized for navigation and positioning. To carry out the field tests, a USRP based SDR receiver is presented, which includes the coarse synchronization and the pilot detection, the first path acquisition and the delay tracking. Field testing campaigns are carried out in five cities in Southern France. The tracking results from the field tests showed the high accuracy of the TOA estimation. Of all the tracking tests, the interval of $95 \%$ accuracy are within $4 \mathrm{~m}$, while the corresponding $C / N_{0}$ varies from 48 to $62 \mathrm{~dB}-\mathrm{Hz}$. In the test with high $C / N_{0}$ condition, the $2 \sigma$ error interval is able to be achieved within 1 meter for a duration 
of $20 \mathrm{~s}$. With the developed TOA estimation method, the synchronization of the emitters is also checked. Testing results showed that the transmitted signals are synchronized with the GPS time, except for one location (signals from Toulouse local emitter) out of the total six investigated locations. Multiple emitters within SFN are also observed during the tests. Meanwhile, the inconsistency between the geometric distance difference and the field tests of the multiple emitters is found and discussed. More investigations on such inconsistency will be taken into account for the future TDOA based positioning with DVB-T signals.

\section{APPENDIX}

We consider an AWGN (additive white Gaussian noise) channel with a high enough SNR. The early cross-correlation branch output in such scenarios can be written as

$$
\left.R_{i, e}(\epsilon)=A e^{j \pi \epsilon} \operatorname{sinc}(\pi \beta(\epsilon-\xi))\right)+n_{e}
$$

where

$$
n_{e}=\frac{1}{N_{p}} \sum_{p \in \mathcal{P}_{s}} c_{i}(p) \cdot e^{j \frac{2 \pi p \xi}{N}} n_{i}(p)
$$

where $n_{i}(p)$ is a while complex Gaussian noise with variance $\frac{\sigma^{2}}{N}$. It can be derived that in (29), $n_{e}$ is a discrete-time independent identically distributed (i.i.d) complex Gaussian process with zero mean and the variance $\sigma_{n_{e}}^{2}=\frac{A \sigma^{2}}{N_{p} \cdot N}$.

The mean of $\left|R_{i, e}(\epsilon)\right|^{2}$ is

$$
\left.\mathrm{E}\left[\left|R_{i, e}(\epsilon)\right|^{2}\right]=A^{2} \operatorname{sinc}^{2}(\pi \beta(\epsilon-\xi))\right)+\sigma_{n_{e}}^{2}
$$

Similarly, by replacing $\xi$ with $-\xi$ in (28)-(30), the output of the late cross-correlation $R_{i, l}(\epsilon)$, the mean and variance of $\left|R_{i, l}(\epsilon)\right|^{2}$ can also be derived.

Therefore, the unnormalized tracking errors are generated as:

$$
a_{i}^{\prime}(\epsilon)=\left|R_{i, e}(\epsilon)\right|^{2}-\left|R_{i, l}(\epsilon)\right|^{2}
$$

Define S-curve $S(\epsilon, \xi)$ as the mean of $a_{i}^{\prime}(\epsilon)$, i.e.,

$$
S(\epsilon, \xi)=\mathrm{E}\left[a_{i}^{\prime}(\epsilon)\right]=A^{2}\left(\operatorname{sinc}^{2}(\pi \beta(\epsilon-\xi))-\operatorname{sinc}^{2}(\pi \beta(\epsilon+\xi))\right)
$$

To get a normalized S-curve, the normalization factor $k_{d}$ in (23) is derived as:

$$
\begin{aligned}
k_{d} & =\left.\frac{\partial S(\epsilon, \xi)}{\partial \epsilon}\right|_{\epsilon=0} \\
& =\frac{2 A^{2}}{\pi^{2} \beta^{2} \xi^{3}}[\pi \beta \xi \sin (2 \pi \beta \xi)+\cos (2 \pi \beta \xi)-1]
\end{aligned}
$$


The research is partially supported by Academy of Finland (grant no.254232). Liang Chen would like to thank Jing Zhou from ISAE-Supaero, France, for the assistance in the outdoor testing campaigns.

\section{REFERENCES}

[1] Y. Wu, S. Hirakawa, U. H. Reimers, and J. Whitaker, "Overview of digital television development worldwide," Proceedings of the IEEE, vol. 94, no. 1, pp. 8-21, Jan 2006.

[2] M. Rabinowitz and J. Spilker, J.J., "A new positioning system using television synchronization signals," IEEE Transactions on Broadcasting, vol. 51, no. 1, pp. 51 - 61, march 2005.

[3] J. T. Ong, H. Yan, S. V. Rao, and G. Shanmugam, "Indoor DTV reception: measurement techniques," IEEE Transactions on Broadcasting, vol. 50, pp. 192-199, 2004.

[4] X. Wang, Y. Wu, and B. Caron, "Transmitter identification using embedded pseudo random sequences," IEEE Transactions on Broadcasting, vol. 50, no. 3, pp. 244-252, September 2004.

[5] X. Wang, Y. Wu, and J. Chouinard, "A new position location system using DTV transmitter identification watermark signals," EURASIP Journal on Applied Signal Processing, vol. 2006, pp. 1-11, 2006.

[6] P. Thevenon, O. Julien, C. Macabiau, D. Serant, L. Ries, S. Corazza, and M. L. Boucheret, "Positioning principles with a mobile TV system using DVB-SH signals and a single frequency network," in Proc. Digital Signal Processing, (DSP09), Santorini, Greece, 5-7 July 2009.

[7] D. Serant, P. Thevenon, M. L. Boucheret, O. Julien, C. Macabiau, S. Corazza, M. Dervin, and L. Ries, "Development and validation of an OFDM/DVB-T sensor for positioning," in Proc. IEEE/ION Position Location and Navigation Symposium (PLANS10), IndianWells, USA, 4-6 May 2010, pp. 998-1001.

[8] D. Serant, O. Julien, C. Macabiau, L. Ries, P. Thevenon, M. Dervin, and M. L. Boucheret, "Positioning using OFDMbased digital TV: new algorithms and tests with real signals," in Proc. 24th International Technical Meeting of the Satellite Division of The Institute of Navigation, Portland OR, USA, 19-23 September 2011, pp. 3451-3460.

[9] D. Serant, O. Julien, L. Ries, P. Thevenon, and M. Dervin, “The digital tv case: Positioning using signals-of-opportunity based on ofdm modulation," Inside GNSS, pp. 54-62, 2011.

[10] L. Dai, Z. Wang, C. Pan, and S. Chen, "Wireless positioning using TDS-OFDM signals in single-frequency networks," IEEE Transactions on Broadcasting, vol. 58, no. 2, pp. 236-246, May 2012.

[11] J. Yang, X. Wang, M. Rahman, S. I. Park, H.-M. Kim, and Y. Wu, "A new positioning system using DVB-T2 transmitter signature waveforms in single frequency networks," IEEE Transactions on Broadcasting, vol. 58, no. 3, pp. 347-359, 2012.

[12] L. Chen, L.-L. Yang, and R. Chen, “Time delay tracking for positioning in DTV networks," in Proc. Ubiquitous Positioning, Indoor Navigation, and Location Based Service (UPINLBS), 2012, Oct. 2012, pp. 1-4.

[13] J. Yan and L. Wu, "A passive location system for single frequency networks using digital terrestrial TV signals," European Transactions on Telecommunications, vol. 22, no. 8, pp. 487-499, 2011.

[14] J. Huang, L. Lo Presti, and R. Garello, "Digital video broadcast-terrestrial (DVB-T) single frequency networks positioning in dynamic scenarios," Sensors, vol. 13, pp. 10 191-10218, 2013.

[15] L. Chen, R. Piché, H. Kuusniemi, and R. Chen, "Adaptive mobile tracking in unknown non-line-of-sight conditions with application to digital TV networks," EURASIP Journal on Advances in Signal Processing, vol. 2014, no. 1, pp. 1-22, 2014. [Online]. Available: http://asp.eurasipjournals.com/content/2014/1/22

[16] M. Rabinowitz and J. Spilker, "Positioning using the ATSC digital television signal," U.S.A. Patent WO: 2004/057 360 A2, 2004-08-07. 
[17] ETSI Standard, ETSI EN 302744 V1.1.4. (2011) Std. ETSI EN 302744 V1.1.4., January 2001.

[18] J. J. van de Beek, M. Sandell, and P. O. Börjesson, "ML estimation of time and frequency offset in OFDM systems," IEEE Tranactions on Signal Processing, vol. 45, pp. 1800-1805, 1997.

[19] B. Yang, K. B. Lataief, R. S. Cheng, and Z. Cao, “Timing recovery for OFDM systems," IEEE Journal on Selected Area in Communications, vol. 18, pp. 2278-2291, November 2000.

[20] A. Mattsson, "Single frequency networks in DTV," IEEE Transactions on Broadcasting, vol. 51, no. 4, pp. 413-422, December 2005.

[21] M. Morelli, C.-C. Kuo, and M.-O. Pun, "Synchronization techniques for orthogonal frequency division multiple access (OFDMA): A tutorial review," Proceedings of the IEEE, vol. 95, no. 7, pp. 1394-1427, July 2007.

[22] T.-D. Chiueh and P.-Y. Tsai, OFDM Baseband Receiver Design for Wireless Communications, 1st ed. Wiley, December 2007.

[23] S. H. Mller-Weinfurtner, J. F. Rler, and J. B. Huber, "Analysis of a frame and frequency synchronizer for (bursty) OFDM,” in Proc. Globecom98 Commun. Theory Mini-Conf., 1998.

[24] S. F. Cotter and B. D. Rao, "Sparse channel estimation via matching pursuit with application to equalization,", IEEE Transactions on Communications, vol. 50, no. 3, pp. 374-377, 2002.

[25] W. Li and J. C. Preisig, "Estimation of rapidly time-varying sparse channels," IEEE Journal of Oceanic Engineering, vol. 32, no. 4, pp. 927-939, Oct. 2007.

[26] E. D. Kaplan, Ed., Understanding GPS: Principles and Applications. Norwood: Artech House, 1996.

[27] "http://www.ettus.com/."

[28] "https://www.ettus.com/content/files/gpsdokit4.pdf."

[29] B. W. Parkinson and J. J. S. Jr., Eds., Gobal Positioning System: Theory and Applications, ser. Progress in Astronautics and Aeronaurtics, vol. 163. Washington DC: AIAA, 1996, vol. I.

[30] "tvignaud.pagesperso-orange.fr/tv/tnt.pdf."

[31] DVB Mega-Frame for Single Frequency Network (SFN) Synchronization, Digital Video Broadcasting (DVB), ETSI Std. TS 101 191, Rev. 1.1.1, April 1997. 